\title{
Asymptotic Law of the $j$ th Records in the Bivariate Exponential Case
}

\begin{abstract}
Grine Azedine
Department of Mathematics, College of Science, Al-Imam Muhammad Ibn Saud Islamic University, P.O. Box 90950, Riyadh 11623, Saudi Arabia
\end{abstract}

Correspondence should be addressed to Grine Azedine; azegri@msn.com

Received 20 February 2014; Accepted 15 June 2014; Published 8 July 2014

Academic Editor: Krassimir T. Atanassov

Copyright (C) 2014 Grine Azedine. This is an open access article distributed under the Creative Commons Attribution License, which permits unrestricted use, distribution, and reproduction in any medium, provided the original work is properly cited.

We consider a sequence $\left(X_{i}, Y_{i}\right)_{1 \leqslant i \leqslant n}$ of independent and identically distributed random variables with joint cumulative distribution $H(x, y)$, which has exponential marginals $F(x)$ and $G(y)$ with parameter $\lambda=1$. We also assume that $X_{i}(\omega) \neq Y_{i}(\omega), \forall i \in \mathbb{N}$, and $\omega \in \Omega$. We denote $\left\{R_{k}^{(j)}\right\}_{k \geqslant 1}$ and $\left\{S_{k}^{(j)}\right\}_{k \geqslant 1}$ by the sequences of the $j$ th records in the sequences $\left(X_{i}\right)_{1 \leqslant i \leqslant n},\left(Y_{i}\right)_{1 \leqslant i \leqslant n}$, respectively. The main result of of the paper is to prove the asymptotic independence of $\left\{R_{k}^{(j)}\right\}_{k \geqslant 1}$ and $\left\{S_{k}^{(j)}\right\}_{k \geqslant 1}$ using the property of stopping time of the $j$ th record times and that of the exponential distribution.

\section{Introduction}

Let $\left(X_{i}\right)_{1 \leqslant i \leqslant n}$ be a sequence of independent and identically distributed (i.i.d) random variables ( $r$.v's.) from a distribution $F$. Let us consider the $j$ th record time defined recurrently for $k=2,3, \ldots$ and $j \geqslant 1$ as

$$
\begin{gathered}
L_{1}^{(j)}=j, \\
L_{k}^{(j)}=\min \left\{m>L_{k-1}^{(j)} ; X_{m-j+1, m}>X_{L_{k-1}^{(j)}-j+1, L_{k-1}^{(j)}}\right\},
\end{gathered}
$$

and the $k$ th ordinary $j$ th record from $F$ as

$$
R_{k}^{(j)}=X_{L_{k}^{(j)}-j+1, L_{k}^{(j)}} ; \quad R_{1}^{(j)}=X_{1, j},
$$

where $X_{i, m}$ denotes the $i$ th order statistic of a sample of size $m$.

In 1976 Dziubdziela-Kopocinski [1] proved that

$$
\lim _{n \rightarrow \infty} \mathbb{P}\left(R_{n}^{(j)} \leqslant a_{n} x+b_{n}\right)=N(-\log (-\log \Theta(x))),
$$

where $\Theta(x)$ is one of the three well known limit laws of $X_{n, n}=\max _{1 \leqslant i \leqslant n}\left(X_{i}\right)$ (Frechet, Wiebull, and Gumbell), $N(\cdot)$ is the standard normal distribution, and $a_{n}, b_{n}$ are the constants of normalization.
Taking account of these three limit laws we get those of the $j$ th record; namely,

(i) Type 1:

$$
N(x), \quad x \in \mathbb{R}
$$

(ii) Type 2:

$$
N_{1 \alpha}= \begin{cases}N\left(\log x^{\alpha}\right), & x>0, \alpha>0, \\ 0 & x \leqslant 0,\end{cases}
$$

(iii) Type 3:

$$
N_{2 \alpha}= \begin{cases}N\left(\log (-x)^{-\alpha}\right), & x<0, \alpha>0, \\ 1 & x \geqslant 0 .\end{cases}
$$

The authors have presented the expressions of the probability density function $f_{n}^{(j)}(x)$ and the distribution function $F_{n}^{(j)}(x)$ of the $j$ th record as follows:

$$
\begin{array}{r}
f_{n}^{(j)}(x)=\frac{j}{(n-1) !}[-j \log (1-F(x))]^{n-1}[1-F(x)]^{j-1} f(x), \\
n=1,2, \ldots, \\
F_{n}^{(j)}(y)=\int_{0}^{-j \log (1-F(y))} e^{-u} \frac{u^{n-1}}{(n-1) !} d u, \quad n=1,2, \ldots
\end{array}
$$


Contrary to that of records, the theory of limit law of the maximum has been extended to the bivariate case by the works of Finkelshtein [2], Geffroy [3], de Oliveira [4], Sibuya [5], Galombos [6], Marshall and Olkin [7], Kotz and Nadarajah [8], Coles [9], and Smith [10].

In this work, we assume that $X_{n}(\omega) \neq Y_{n}(\omega), \forall n \in \mathbb{N}$ and $\omega \in \Omega$. Moreover, we will focus on the bivariate $r . v^{\prime} s .\left(R_{n}^{(j)}, S_{n}^{(j)}\right)_{n \geqslant 1}$ issued from pairs of r.v's. $\left(X_{n}, Y_{n}\right)_{n \geqslant 1}$ with joint cumulative distribution $H(x, y)$ whose marginals $F(x)$ and $G(y)$ are exponential with parameter $\lambda=1$ and prove that

$$
\begin{aligned}
\lim _{n \rightarrow \infty} \mathbb{P}\left(R_{n}^{(j)} \leqslant a_{n} x+b_{n}, S_{n}^{(j)} \leq c_{n} y+d_{n}\right) \\
=\lim _{n \rightarrow \infty} \mathbb{P}\left(R_{n}^{(j)} \leqslant a_{n} x+b_{n}\right) \lim _{n \rightarrow \infty} \mathbb{P}\left(S_{n}^{(j)} \leqslant c_{n} y+d_{n}\right) \\
=N(x) N(y),
\end{aligned}
$$

where $N(\cdot)$ is the standard normal distribution, and $a_{n}=c_{n}=$ $n / j, b_{n}=d_{n}=\sqrt{n / j^{2}}$.

\section{Preliminaries}

In this section we recall some relevant results for future use.

Theorem 1 (Deheuvels [11]). Let $X_{1}, X_{2}, \ldots, X_{n}$ be a sequence of i.i.d r.v's. with $\Phi(x)$ continues repartition function.

Let $\left\{X_{k, n}: 1 \leqslant k \leqslant n\right\}$ and $\left\{R_{k}^{(j)}, k \geqslant 1\right\}$ be, respectively, the sequences of order statistics and $j$ th records.

$\forall n \geqslant 2$ and for all $j \geqslant 1$ one has the following.

(i) For $n \geqslant j \geqslant 1$ and $\Phi(s)<1$, the sequences

$$
\left\{X_{k, n}: 1 \leq k \leq n\right\}, \quad\left\{R_{k}^{(j)}: k \geqslant 1\right\}
$$

form a Markov chain with equal probability of transition. That means

$$
\begin{aligned}
\mathbb{P}\left(R_{k+1}^{(j)} \geqslant t \mid R_{k}^{(j)}=s\right) & =\mathbb{P}\left(X_{n-j+1, n} \geqslant t \mid X_{n-j, n}=s\right) \\
& =\left(\frac{1-\Phi(t)}{1-\Phi(s)}\right)^{j} .
\end{aligned}
$$

(ii) The assertions $\left\{R_{k}^{(j)}\right.$ and $R_{k+1}^{(j)}-R_{k}^{(j)}$ independent $\}$ and $\left\{X_{n-j, n}\right.$ and $X_{n-j+1, n}-X_{n-j, n}$ independent $\}$ are equivalent.

Corollary 2. Let $\left(X_{i}\right)_{1 \leqslant i \leqslant n}$ be a sequence of i.i.d r.v's. with repartition $\Phi(x)$ continues and let, for $j \geqslant 1$ fixed, $\left(R_{k}^{(j)}\right)_{k \geqslant 1}$ be the sequence of $j$ th records from $\Phi$.

If $k \geqslant 1$ is a fixed integer, $R_{k}^{(j)}$ and $R_{k+1}^{(j)}-R_{k}^{(j)}$ are independent if and only if $\Phi(x)=1-e^{(-b(x-B))}$ with $x \geqslant B$ for $B$ and $b$ being positive finite constants.

Corollary 3. For $\Phi(x)=1-e^{-x}, x \geqslant 0$ and $\left(R_{k}^{(j)}\right)_{k \geqslant 1}$ being the sequence of $j$ th records, then $\alpha_{k}^{(j)}=\left(R_{k}^{(j)}-R_{k-1}^{(j)}\right)_{1 \leqslant k \leqslant n}$ is a sequence of i.i.d r.v's.s with repartition function $\Psi(x)=1-$ $e^{-j x}, x \geqslant 0$.
Corollary 4. For $\Phi(x)=1-e^{-x}, x \geqslant 0$, and for all $m \leq k-1$, $R_{k}^{(j)}-R_{k-1}^{(j)}$ is independent of $R_{m}$.

Let $\left(U_{i}\right)_{1 \leqslant i \leqslant n}$ be the sequence of i.i.d r.v's. uniformly distributed on $[0,1]$; then one has the following.

Theorem 5 (Deheuvels [12]). For all $A>0$ and for all $P \geqslant$ 4 a.s. for $n \rightarrow \infty$,

$$
\begin{aligned}
& U_{m, n} \\
& \leqslant \frac{1}{n}\left\{\log _{2} n+(m+1) \log _{3} n+\log _{4} n+\cdots+(1+A) \log _{p} n\right\} .
\end{aligned}
$$

Theorem 6 (Deheuvels [12]). For all $A>0$, and for all $p \geqslant$ 3 a.s. for $n$ large enough,

$$
U_{m, n}>\frac{1}{n\left\{(\log n)\left(\log _{2} n\right) \cdots\left(\log _{p} n\right)^{1+A}\right\}^{1 / m}} .
$$

Corollary 7. Let $X_{1}, X_{2}, \ldots, X_{n}$ be a sequence of i.i.d r.v's. with repartition function $\Phi(x)$ and $\Gamma(t)=\inf \{x \in \mathbb{R} \mid \Phi(x) \geq$ t\}. Then, a.s. for $n \rightarrow \infty, A>0, p \geqslant 4$,

$$
\begin{gathered}
\Gamma\left\{\frac{1}{n\left\{(\log (n))\left(\log _{2}(n)\right) \cdots\left(\log _{p}(n)\right)^{1+A}\right\}^{1 / m}}\right\} \\
\leqslant X_{m, n} \\
\leqslant \Gamma\left\{\frac { 1 } { n } \left\{\log _{2}(n)+(m+1) \log _{3}(n)\right.\right. \\
\left.\left.+\cdots+(1+A) \log _{p}(n)\right\}\right\} .
\end{gathered}
$$

Moreover, a.s., for $A=0$, both members of these two inequalities are not satisfied infinitely many times.

Corollary 8. If $\Phi(x)=1-e^{-x}, x>0$, then $\Gamma(x)=-\log (1-$ $x), x<1$.

Theorem 9 (Barndorff-Nielsen [13]). If

$$
\begin{gathered}
\lim _{n \rightarrow \infty} \mathbb{P}\left\{X_{m, n} \leqslant \lambda_{n}\right\}=0, \\
\sum_{n} n^{m-1}\left\{\Phi\left(\lambda_{n}\right)\right\}^{n-m+1}\left\{1-\Phi\left(\lambda_{n+1}\right)\right\}^{m}<\infty,
\end{gathered}
$$

then $\lambda_{n} \ll X_{m, n}$ a.s. with $\left\{\lambda_{n}\right\}_{n \geqslant 1}$ an increasing sequence of numbers.

Lemma 10. If $\left(X_{i}, Y_{i}\right)_{1 \leqslant i \leqslant n}$ is a sequence of i.i.d pairs of r.v's. with joint cumulative distribution function $\Phi(x, y)$, then

$$
\Phi(x, y)=\Phi_{X}(x)+\Phi_{Y}(y)+\mathbb{P}(X>x, Y>y)-1 .
$$

Proof. The proof is obvious. 
Theorem 11 (Marshall and Olkin [7]). If

$$
\begin{aligned}
& \Phi_{X}(x)=1-e^{-x}, \quad x>0, \\
& \Phi_{Y}(y)=1-e^{-y}, \quad y>0,
\end{aligned}
$$

then $\mathbb{P}(X>x, Y>y)=e^{-x-y-\lambda \max (x, y)}, \lambda>0$.

Theorem 12 (Galombos [6]). Let $\left(u_{n}\right)_{n \geq 1}$ and $\left(v_{n}\right)_{n \geq 1}$ be two sequences of r.v's.; suppose that there exists a distribution function $\Pi(x)$ such that at any point $x$ of continuity of $\Pi$

$$
\mathbb{P}\left(u_{n} \leqslant x\right) \underset{n \rightarrow \infty}{\longrightarrow} \Pi(x) .
$$

Suppose that for all $\epsilon>0$

$$
\mathbb{P}\left(\left|v_{n}\right|>\epsilon\right) \underset{n \rightarrow \infty}{\longrightarrow} 0 .
$$

Then $\lim _{n \rightarrow \infty} \mathbb{P}\left(u_{n}+v_{n}<x\right)=\Pi(x)$ at all points of continuity of $\Pi$.

\section{Main Result}

Throughout the following, we consider $\left(X_{i}, Y_{i}\right)_{1 \leqslant i \leqslant n}$ as a sequence of i.i.d r.v's. with joint cumulative distribution function $H(x, y)$ whose marginals are, respectively, $F(x)=$ $1-e^{-x}, x>0$ and $G(y)=1-e^{-y}, y>0$.

3.1. Study of the Number of Coincidences. Let $\left\{n_{k}^{(j)}\right\}_{k \geqslant 1}$ and $\left\{m_{k}^{(j)}\right\}_{k \geqslant 1}$ be the sequences of $j$ th record times associated, respectively, to $X_{i}$ and $Y_{i}$, with $R_{1}^{(j)}=X_{1, j}$ and $S_{1}^{(j)}=Y_{1, j}$ dependent, suggesting that every time $n_{k}^{(j)}=m_{k^{\prime}}^{(j)}$, for any $k$ and $k^{\prime}$, there is dependence of the $j$ th corresponding records.

Lemma 13. The number of coincidences, which means the number of times $n_{k}^{(j)}$ and $m_{k}^{(j)}$ belong, respectively, to the sets $\left\{m_{1}^{(j)}, m_{2}^{(j)}, \ldots, m_{k}^{(j)}, \ldots\right\}$ and $\left\{n_{1}^{(j)}, n_{2}^{(j)}, \ldots, n_{k}^{(j)}, \ldots\right\}$, is finite.

Proof. Let us study the nature of the following series

$$
\begin{aligned}
\sum_{n} \mathbb{P} & \left(A_{n}\right) \\
& =\sum_{n} \mathbb{P}\left\{X_{n-j+1, n}>X_{n-j, n-1} ; Y_{n-j+1, n}>Y_{n-j, n-1}\right\},
\end{aligned}
$$

where $A_{n}$ is the event for which $n_{k}^{(j)}=m_{k^{\prime}}^{(j)}=n$.

As $\left\{X_{n-j+1, n}>X_{n-j, n-1} ; Y_{n-j+1, n}>Y_{n-j, n-1}\right\}$ is equivalent to $\left\{X_{n}>X_{n-j, n-1} ; Y_{n}>Y_{n-j, n-1}\right\}$, studying the nature of the series $\sum_{n} \mathbb{P}\left(A_{n}\right)$ is equivalent to the study of the series $\sum_{n} \mathbb{P}\left(A_{n}^{\prime}\right)$ with

$$
A_{n}^{\prime}=\left\{X_{n}>X_{n-j, n-1} ; Y_{n}>Y_{n-j, n-1}\right\} .
$$

Let $\left(X_{n}\right)_{n \geqslant 1}$ be a sequence of i.i.d r.v's. with repartition $\Phi(x)$ and $\left(U_{n}\right)_{n \geq 1}$ the sequence of i.i.d r.v's. uniformly distributed on $[0,1]$ with

$$
\Gamma(t)=\inf \{x \in \mathbb{R} \mid \Phi(x) \geq t\} .
$$

$\left\{\Gamma\left(U_{n}\right)\right\}_{n \geq 1}$ and $\left(X_{n}\right)_{n \geqslant 1}$ are identically distributed.
An a.s. bounding of $X_{m, n}$ is equivalent to an a.s. bounding of $U_{m, n}$.

Using Theorems 5 and 6, and Corollary 7 of Deheuvels and Theorem 9 of Barndorff-Nielsen we obtain the a.s. bounding of $X_{n-j+1, n}$.

Choosing $\lambda_{n}=1-\left(1 / n(\log n)^{(1+\epsilon) /(n-j+1)}\right), \epsilon>0$, we obtain a lower bound of $U_{n-j+1}$.

Indeed

$$
\begin{gathered}
F_{U}\left(\lambda_{n}\right)=1-\frac{1}{n(\log n)^{(1+\epsilon) /(n-j+1)}}, \\
\sum_{n} n^{m-1}\left(F_{U}\left(\lambda_{n}\right)\right)^{n-m+1}\left(1-F_{U}\left(\lambda_{n+1}\right)\right)^{m} \\
=\sum_{n} n^{n-j}\left(1-\frac{1}{n(\log n)^{(1+\epsilon) /(n-j+2)}}\right)^{j} \\
\times\left(\frac{1}{(n+1)(\log n+1)^{(1+\epsilon) /(n-j+2)}}\right)^{n-j+1} .
\end{gathered}
$$

Let $S_{n}$ be the general term of the series in the right side of the above equality. It is clear that

$$
S_{n} \leqslant V_{n}
$$

with

$$
\begin{aligned}
V_{n} & =\frac{n^{n-j}}{n^{n-j+1}} \frac{1}{(\log n)^{(1+\epsilon)((n-j+1) /(n-j+2))}} \\
& =\frac{1}{n} \frac{1}{(\log n)^{(1+\epsilon)((n-j+1) /(n-j+2))}} .
\end{aligned}
$$

For $n$ large enough $V_{n} \sim W_{n}$ with $W_{n}=1 / n(\log n)^{1+\epsilon}$ is a general term of Bertrand convergent series.

Hence we get

$$
\sum_{n} S_{n} \leqslant+\infty
$$

which implies that

$$
U_{n-j+1, n}>1-\frac{1}{n(\log n)^{(1+\epsilon) /(n-j+1)}} .
$$

Consequently, we have

$$
X_{n-j+1, n}>\Gamma\left(\lambda_{n}\right)
$$

Using Corollary 8, we get $\Gamma\left(\lambda_{n}\right)=-\log \left(1-\lambda_{n}\right)=$ $\log \left\{n(\log n)^{(1+\epsilon) /(n-j+1)}\right\}$.

Since

$$
\left\{X_{n}>X_{n-j, n-1}, Y_{n}>Y_{n-j, n-1}\right\}
$$

implies

$$
\left\{X_{n}>\Gamma\left(\lambda_{n}\right), Y_{n}>\Gamma\left(\lambda_{n}\right)\right\},
$$


it suffices to study the nature of the series $\sum_{n} \mathbb{P}\left(A_{n}^{\prime \prime}\right)$ to know that of $\sum_{n} \mathbb{P}\left(A_{n}^{\prime}\right)$, where $A_{n}^{\prime \prime}$ is given by

$$
\begin{aligned}
A_{n}^{\prime \prime}=\left\{X_{n}>\log \left(n(\log n)^{(1+\epsilon) /(n-j+1)}\right) ;\right. \\
\left.Y_{n}>\log \left(n(\log n)^{(1+\epsilon) /(n-j+1)}\right)\right\} .
\end{aligned}
$$

Using Theorem 11 of Marshall and Olkin, we get

$$
\begin{aligned}
\mathbb{P} & \left(X_{n}>\Gamma\left(\lambda_{n}\right), Y_{n}>\Gamma\left(\lambda_{n}\right)\right) \\
& =\frac{1}{n^{2+\lambda}(\log n)^{(1+\epsilon)((2+\lambda) /(n-j+1))}} \\
& =K_{n} \leqslant K_{n}^{\prime}=\frac{1}{n^{2+\lambda}},
\end{aligned}
$$

with $\sum_{n} K_{n}^{\prime}<\infty$ (Riemann's series).

Then

$$
\begin{aligned}
\sum_{n} K_{n}^{\prime}<\infty & \Longrightarrow \sum_{n} K_{n}<\infty \Longrightarrow \sum_{n} \mathbb{P}\left(A_{n}^{\prime \prime}\right) \\
<\infty & \Longrightarrow \sum_{n} \mathbb{P}\left(A_{n}^{\prime}\right)<\infty \Longrightarrow \sum_{n} \mathbb{P}\left(A_{n}\right)<\infty .
\end{aligned}
$$

By Borel-Cantelli's Lemma,

$$
\sum_{n} \mathbb{P}\left(A_{n}\right)<\infty \Longrightarrow \varlimsup_{n} A_{n}=0,
$$

which means that with probability one at most a finite number of $A_{n}$ are realized simultaneously and consequently that the number of coincidences is finite a.s.

3.2. Some Record Series. Let us consider and give some properties of the following sequences:

$$
\begin{gathered}
R_{k}^{(j)}=\sum_{i=1}^{k} \alpha_{i}^{(j)} \quad \text { with } \quad \alpha_{i}^{(j)}=R_{i+1}^{(j)}-R_{i}^{(j)} \\
S_{k}^{(j)}=\sum_{i=1}^{k} \beta_{i}^{(j)} \quad \text { with } \quad \beta_{i}^{(j)}=S_{i+1}^{(j)}-S_{i}^{(j)} \\
R_{k}^{*(j)}=\sum_{i=1}^{n} \alpha_{i}^{(j)} \gamma_{i} \\
\text { with } \quad \gamma_{i}= \begin{cases}1 & \text { if } n_{i}^{(j)} \notin\left\{m_{1}^{(j)}, \ldots, m_{k}^{(j)}, \ldots\right\}, \\
0 & \text { otherwise }\end{cases} \\
S_{k}^{*(j)}=\sum_{i=1}^{n} \beta_{i}^{(j)} \delta_{i} \\
\text { with } \quad \delta_{i}= \begin{cases}1 & \text { if } m_{i}^{(j)} \notin\left\{n_{1}^{(j)}, \ldots, n_{k}^{(j)}, \ldots\right\}, \\
0 & \text { otherwise. }\end{cases}
\end{gathered}
$$

Lemma 14. Consider the following

$$
R_{n}^{(j)}-R_{n}^{*(j)} \underset{n \rightarrow \infty}{\longrightarrow} C_{1}<\infty \quad \text { a.s. }
$$

Proof. Consider the following

$$
\begin{aligned}
R_{n}^{(j)}-R_{n}^{*(j)} & =\sum_{i=1}^{n} \alpha_{i}^{(j)}-\sum_{i=1}^{n} \alpha^{(j)} \gamma_{i} \\
& =\sum_{i=1}^{n}\left(1-\gamma_{i}\right) \alpha_{i}^{(j)} \\
& =\sum_{i=1}^{n} \theta_{i} \alpha_{i}^{(j)}
\end{aligned}
$$

with

$$
\theta_{i}= \begin{cases}1 & \text { if } n_{i}^{(j)} \in\left\{m_{1}^{(j)}, \ldots, m_{k}^{(j)}, \ldots\right\} \\ 0 & \text { otherwise. }\end{cases}
$$

Hence

$$
R_{n}^{(j)}-R_{n}^{*(j)}=\sum_{i \in I} \alpha_{i}^{(j)}
$$

where $I$ is the set of $i$ for which there are coincidences; as this set is finite $\sum_{i \in I} \alpha_{i}^{(j)}<\infty$. By the same method we have

$$
S_{n}^{(j)}-S_{n}^{*(j)} \underset{n \rightarrow \infty}{\longrightarrow} C_{2}<\infty \quad \text { a.s. }
$$

Lemma 15. Consider

$$
\frac{\left(R_{n}^{*(j)}-(n / j)\right)}{\sqrt{n / j^{2}}} \underset{n \rightarrow \infty}{\stackrel{\mathscr{L}}{\longrightarrow}} N(0,1) .
$$

Proof. Consider the following

$$
\begin{aligned}
\frac{R_{n}^{*(j)}-(n / j)}{\sqrt{n / j^{2}}} & =\frac{R_{n}^{*(j)}-R_{n}^{(j)}+R_{n}^{(j)}-(j / n)}{\sqrt{n / j^{2}}} \\
& =\frac{R_{n}^{*(j)}-R_{n}^{(j)}}{\sqrt{n / j^{2}}}+\frac{R_{n}^{(j)}-(n / j)}{\sqrt{n / j^{2}}} .
\end{aligned}
$$

Using Corollary 3 and Theorem 12 of Galombos and the central limit theorem we get

$$
\begin{aligned}
& \lim _{n \rightarrow \infty} \mathbb{P}\left\{\frac{R_{n}^{*(j)}-(n / j)}{\sqrt{n / j^{2}}} \leqslant x\right\} \\
& =\lim _{n \rightarrow \infty} \mathbb{P}\left\{\frac{R_{n}^{*(j)}-R_{n}^{(j)}+R_{n}^{(j)}-(j / n)}{\sqrt{n / j^{2}}} \leqslant x\right\}
\end{aligned}
$$




$$
\begin{aligned}
& =\lim _{n \rightarrow \infty} \mathbb{P}\left\{\frac{R_{n}^{*(j)}-R_{n}^{(j)}}{\sqrt{n / j^{2}}}+\frac{R_{n}^{(j)}-(n / j)}{\sqrt{n / j^{2}}} \leqslant x\right\} \\
& =\lim _{n \rightarrow \infty} \mathbb{P}\left\{\frac{R_{n}^{(j)}-(n / j)}{\sqrt{n / j^{2}}} \leqslant x\right\} \\
& =\frac{1}{\sqrt{2 \Pi}} \int_{0}^{x} e^{-u^{2} / 2} \mathrm{~d} u \\
& =N(x) .
\end{aligned}
$$

By the same method, we have

$$
\frac{S_{n}^{*(j)}-(n / j)}{\sqrt{n / j^{2}}} \underset{n \rightarrow \infty}{\stackrel{\mathscr{L}}{\longrightarrow}} N(0,1) .
$$

Now, let us consider the two following sequences:

$$
R_{n}^{* *(j)}=\sum_{k=1}^{N(n)} \alpha_{k}^{(j)} \gamma_{k}, \quad S_{n}^{* *(j)}=\sum_{k=1}^{M(n)} \beta_{k}^{(j)} \delta_{k}
$$

with

$$
\begin{aligned}
& N(n)=\inf \left\{m \geqslant 1 \mid \sum_{k=1}^{m} \gamma_{k}=n\right\}, \\
& M(n)=\inf \left\{m \geqslant 1 \mid \sum_{k=1}^{m} \delta_{k}=n\right\} .
\end{aligned}
$$

Lemma 16. Consider the following

$$
R_{n}^{* *(j)}-R_{n}^{*(j)} \underset{n \rightarrow \infty}{\longrightarrow} k_{1}<\infty \quad \text { a.s. }
$$

Proof. Since the number of coincidences is finite, there exists $n_{0}$ finite, such that, for all $n>n_{0}, \gamma_{n}=1$;

$$
\begin{aligned}
R_{n}^{* *(j)}-R_{n}^{*(j)} & \\
= & \sum_{k=1}^{N(n)} \alpha_{k}^{(j)} \gamma_{k}-\sum_{k=1}^{n} \alpha_{k}^{(j)} \gamma_{k} \\
= & \sum_{k=1}^{n_{0}} \alpha_{k}^{(j)} \gamma_{k}+\sum_{k=n_{0}+1}^{N(n)} \alpha_{k}^{(j)} \gamma_{k} \\
& -\sum_{k=1}^{n_{0}} \alpha_{k}^{(j)} \gamma_{k}-\sum_{k=n_{0}+1}^{n} \alpha_{k}^{(j)} \gamma_{k} \\
= & \sum_{k=n_{0}+1}^{N(n)} \alpha_{k}^{(j)} \gamma_{k}-\sum_{k=n_{0}+1}^{n} \alpha_{k}^{(j)} \gamma_{k} \\
= & \sum_{k=n_{0}+1}^{N(n)} \alpha_{k}^{(j)}-\sum_{k=n_{0}+1}^{n} \alpha_{k}^{(j)}
\end{aligned}
$$

$$
\begin{aligned}
& =\sum_{k=n_{0}+1}^{n} \alpha_{k}^{(j)}+\sum_{k=n+1}^{N(n)} \alpha_{k}^{(j)}-\sum_{k=n_{0}+1}^{n} \alpha_{k}^{(j)} \\
& =\sum_{k=n+1}^{N(n)} \alpha_{k}^{(j)} .
\end{aligned}
$$

$R_{n}^{* *(j)}-R_{n}^{*(j)}$ is the sum of $(N(n)-n)$ i.i.d r.v's.

Between 1 and $n_{0}$ we have $K$ coincidences and $r$ noncoincidences, so we must have $(n-r)$ noncoincidences between $\left(n_{0}+1\right)$ and $N(n)$; hence

$$
N(n)=K+r+n-r=K+n .
$$

This implies that $N(n)-n=K$ with $K$ finite, so $R_{n}^{* *(j)}-R_{n}^{*(j)}$ is a finite sum of $r . v^{\prime}$ 's, which proves our result.

By the same method, we have

$$
S_{n}^{* *(j)}-S_{n}^{*(j)} \underset{n \rightarrow \infty}{\longrightarrow} k_{2}<\infty \quad \text { a.s. }
$$

Lemma 17. $R_{n}^{* *(j)}$ and $S_{n}^{* *(j)}$ are individually the sum of $n$ i.i.d exponential r.v's. with parameter $j$ so by the central limit theorem one gets

$$
\begin{aligned}
& \frac{R_{n}^{* *(j)}-(n / j)}{\sqrt{n / j^{2}}} \underset{n \rightarrow \infty}{\stackrel{\mathscr{L}}{\longrightarrow}} N(0,1), \\
& \frac{S_{n}^{* *(j)}-(n / j)}{\sqrt{n / j^{2}}} \underset{n \rightarrow \infty}{\stackrel{\mathscr{L}}{\longrightarrow}} N(0,1) .
\end{aligned}
$$

\subsection{Study of the jth Record Times}

Definition 18. Let $(\Omega, \mathscr{F})$ be a measurable space and $\left(\mathscr{F}_{t}\right)_{t \in I}$ be an increasing family of sub- $\sigma$-algebra of $\mathscr{F}$, defined on an interval $I$ of $\overline{\mathbb{R}}$ or $\overline{\mathbb{N}}$.

According to $\left(\mathscr{F}_{t}\right)_{t \in I}$ a r.v. $T$ defined on $(\Omega, \mathscr{F})$ with values in $I$ is called a stopping time if

$$
\{\omega: T(\omega) \leqslant t\} \in \mathscr{F}_{t}, \quad \forall t \in I .
$$

Interpretation. The notion of stopping time corresponds to the notion of stopping some process at a random time $T$. Indeed, the stopping time is a function of $\omega$. Also the definition (51) expresses that all the contingencies that lead to the previous stopping time are an event of $\mathscr{F}_{t}$, that means an event definable by the behavior of the phenomenon before time $t$.

Lemma 19. The sequence of $j$ th record times $\left\{n_{k}^{(j)}\right\}_{k \geqslant 1}$ is a sequence of stopping time.

Proof. Considering the sub- $\sigma$-algebra $a_{n}=$ $\sigma\left(X_{1}, Y_{1}, \ldots, X_{n}, Y_{n}\right)$ and $a_{t}=\sigma\left(X_{1}, Y_{1}, \ldots, X_{t}, Y_{t}\right)$ the increasing family of sub- $\sigma$-algebra of $a_{n}, n_{k}^{j}$ is a stopping time if and only if

$$
\left\{n_{k}^{(j)}=t\right\} \in a_{t} \quad \forall t \in \overline{\mathbb{N}} .
$$

But $n_{k}^{(j)}=t$ is equivalent to $X_{n_{k}^{(j)}-j+1, n_{k}^{(j)}}=X_{t-j+1, t} \mathbb{1}_{\left\{n_{k}^{(j)}=t\right\}}$. 
$X_{t-j+1, t}$ is $\sigma\left(X_{1, t}, \ldots, X_{t-j+1, t}\right)$ measurable and included in $\sigma\left(X_{1}, \ldots, X_{t}\right)$ because the ordered statistic is exhaustive.

But $\sigma\left(X_{1}, \ldots, X_{t}\right)$ is included in $\sigma\left(X_{1}, Y_{1}, \ldots, X_{t}, Y_{t}\right)$ which is equal to $a_{t}$.

Consequently

$$
\left\{n_{k}^{(j)}=t\right\} \in a_{t} \quad \forall t \in I \subseteq \overline{\mathbb{N}}
$$

which implies that $n_{k}^{(j)}$ is a stopping time.

By the same method $m_{k}^{(j)}$ is also a stopping time.

3.4. Asymptotic Independence of $R_{n}^{(j)}$ and $S_{n}^{(j)}$. In this section we will prove the asymptotic independence of $\left\{R_{n}^{(j)}\right\}_{n \geqslant 1}$ and $\left\{S_{n}^{(j)}\right\}_{n \geqslant 1}$ sequences of records issued from $\left(X_{i}\right)_{1 \leq i \leq n}$ and $\left(Y_{i}\right)_{1 \leq i \leq n}$, respectively.

Proposition 20. Let $\left(R_{n}^{(j)}, S_{n}^{(j)}\right)_{n \geqslant 1}$ be a bivariate r.v's. of records issued from pairs of r.v's. $\left(X_{n}, Y_{n}\right)_{n \geqslant 1}$ with joint cumulative distribution $H(x, y)$ whose marginals $F(x)$ and $G(y)$ are exponential with parameter $\lambda=1$; then

$$
\begin{aligned}
& \lim _{n \rightarrow \infty} \mathbb{P}\left(\frac{R_{n}^{(j)}-(n / j)}{\sqrt{n / j^{2}}} \leqslant x, \frac{S_{n}^{(j)}-(n / j)}{\sqrt{n / j^{2}}} \leqslant y\right) \\
& =\lim _{n \rightarrow \infty} \mathbb{P}\left(\frac{R_{n}^{(j)}-(n / j)}{\sqrt{n / j^{2}}} \leqslant x\right) \lim _{n \rightarrow \infty} \mathbb{P}\left(\frac{S_{n}^{(j)}-(n / j)}{\sqrt{n / j^{2}}} \leqslant y\right) \\
& =N(x) N(y),
\end{aligned}
$$

where $N(\cdot)$ is the standard normal distribution.

Proof. Since there exists an $n_{0}$ from which there is no coincidences, throughout the following, we will consider the case where we observe, first, a record $R_{k}^{(j)}$ for the $X_{i}$.

$a_{n_{k}^{(j)}}=\left\{A \mid A \cap n_{k}^{(j)}=t \in a_{t} \forall t \in I \subseteq \overline{\mathbb{N}}\right\}$ being the algebra of events prior $n^{(j)}$, all that has led to the appearance of $R_{k}^{(j)}$ that depends only on what has been observed before $n_{k}^{(j)}$.

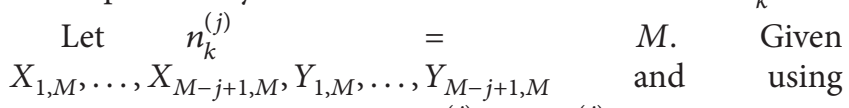
Corollary 4, the first record $S_{i}^{(j)}$ with $n_{i}^{(j)}>M$ is such that $S_{i}^{(j)}-S_{i-1}^{(j)}$ is independent of $Y_{1, M}, \ldots, Y_{M-j+1, M}$ and fortiori of $X_{1, M}, \ldots, X_{M-j+1, M}$.

Thus, $\alpha_{i}^{(j)}=R_{i}^{(j)}-R_{i-1}^{(j)}, n_{i}^{(j)}$ and $\beta_{i}^{(j)}=S_{i}^{(j)}-S_{i-1}^{(j)}, m_{i}^{(j)} \geqslant$ $M$ are independent from all what has been observed before $n_{k}^{(j)}=M$, including $R_{k}^{(j)}$, which means it is independent of $a_{M}$.

We conclude that

(i) for all $i$ such that $n_{i}^{(j)} \geqslant n_{k}^{(j)}, R_{i}^{* *(j)}$ is asymptotically independent of $S_{i_{1}}^{* *(j)}$ with $m_{i_{1}}^{(j)}<n_{k}^{(j)}$,

(ii) for all $t$ such that $m_{t}^{(j)} \geqslant n_{k}^{(j)}, S_{t}^{* *(j)}$ is asymptotically independent of $R_{i_{2}}^{* *(j)}$ with $n_{i_{2}}^{(j)}<n_{k}^{(j)}$.
This sequential reasoning will be taken each time we have either a record for the $X_{i}$ or a record for the $Y_{i}$.

Thus, we prove that $R_{n}^{* *(j)}$ and $S_{n}^{* *(j)}$ are asymptotically independent and consequently $R_{n}^{(j)}$ and $S_{n}^{(j)}$ will be as well.

Indeed, if we denote, for the sake of simplicity, $\lim _{n \rightarrow \infty} \mathbb{P}\left(\left(\left(R_{n}^{(j)}-(n / j)\right) / \sqrt{n / j^{2}}\right) \leqslant x,\left(\left(S_{n}^{(j)}-(n / j)\right) / \sqrt{n / j^{2}}\right) \leqslant\right.$ $y$ ) by $\alpha$,we get

$$
\begin{array}{r}
\alpha=\lim _{n \rightarrow \infty} \mathbb{P}\left(\frac{R_{n}^{(j)}-R_{n}^{*(j)}+R_{n}^{*(j)}-R_{n}^{* *(j)}+R_{n}^{* *(j)}-(n / j)}{\sqrt{n / j^{2}}} \leqslant x,\right. \\
\left.\frac{S_{n}^{(j)}-S_{n}^{*(j)}+S_{n}^{*(j)}-S_{n}^{* *(j)}+S_{n}^{* *(j)}-(n / j)}{\sqrt{n / j^{2}}} \leqslant y\right) .
\end{array}
$$

Using (35), (39), and Theorem 12

$$
\begin{array}{r}
\alpha=\lim _{n \rightarrow \infty} \mathbb{P}\left(\frac{R_{n}^{*(j)}-R_{n}^{* *(j)}+R_{n}^{* *(j)}-(n / j)}{\sqrt{n / j^{2}}} \leqslant x,\right. \\
\left.\frac{S_{n}^{*(j)}-S_{n}^{* *(j)}+S_{n}^{* *(j)}-(n / j)}{\sqrt{n / j^{2}}} \leqslant y\right) .
\end{array}
$$

Using (46), (49), and Theorem 12

$$
\alpha=\lim _{n \rightarrow \infty} \mathbb{P}\left(\frac{R_{n}^{* *(j)}-(n / j)}{\sqrt{n / j^{2}}} \leqslant x, \frac{S_{n}^{* *(j)}-(n / j)}{\sqrt{n / j^{2}}} \leqslant y\right) .
$$

Now using (50) we obtain

$$
\begin{aligned}
\alpha= & \lim _{n \rightarrow \infty} \mathbb{P}\left(\frac{R_{n}^{* *(j)}-(n / j)}{\sqrt{n / j^{2}}} \leqslant x\right) \\
& \times \lim _{n \rightarrow \infty} \mathbb{P}\left(\frac{S_{n}^{* *(j)}-(n / j)}{\sqrt{n / j^{2}}} \leqslant y\right) \\
= & N(x) N(y),
\end{aligned}
$$

which proves our result.

\section{Conclusion}

In this paper we proved the asymptotic independence of the $j$ th records $R_{k}^{(j)}$ and $S_{k}^{(j)}$ in the exponential case $(\lambda=1)$, from sequences $\left(X_{i}\right)_{1 \leqslant i \leqslant n}$ and $\left(Y_{i}\right)_{1 \leqslant i \leqslant n}$, respectively. In this case we know that $X_{n, n}=\max _{1 \leqslant i \leqslant n}\left(X_{i}\right)$ and $Y_{n, n}=\max _{1 \leqslant i \leqslant n}\left(Y_{i}\right)$ are the type Gumbell, respectively. It would be interesting to see if we can obtain the same result when $X_{n, n}$ and $Y_{n, n}$ are Frechet or Weibull type. 


\section{Conflict of Interests}

The author declares that there is no conflict of interests regarding the publication of this paper.

\section{References}

[1] Dziubdziela-Kopocinski, "Limiting Properties of the k-th Record," Applicationes Mathematicae, vol. 15, pp. 187-190, 1976.

[2] B. V. Finkelshtein, "On the limiting distributions of the extreme terms of a variational series of a two-dimensional random quantity," Doklady Akademii Nauk SSSR, vol. 91, pp. 209-211, 1953 (Russian).

[3] J. Geffroy, "Contribution a l'etude des valeurs extremes," Pub. Int. Stat. Paris, vol. 7, 37 - 121 and 8, pp. 124-184, 1958.

[4] J. T. de Oliveira, "Structure theory of bivariate extremes, extension," Estudos de Matematica, Estatistica, e Economicos, vol. 7, pp. 165-195, 1962.

[5] M. Sibuya, "Bivariate extremal statistics," Annals of the Institute of Statistical Mathematics, vol. 11, pp. 195-210, 1960.

[6] J. Galombos, The Asymptototic Theory of Extremes Order Statistics, John Wiley \& Sons, New York, NY, USA, 1978.

[7] A. W. Marshall and I. Olkin, "Domains of attraction of multivariate extreme value distributions," The Annals of Probability, vol. 11, no. 1, pp. 168-177, 1983.

[8] K. Kotz and D. Nadarajah, Extreme Valu e Distribution. Theory and Applications, Imperial College Press, London, UK, 2000.

[9] S. G. Coles, Introduction to Statistical Modeling of Extreme Values, Springer, New York, NY, USA, 2001.

[10] R. L. Smith, Extreme Values, Chapman \& Hall, London, UK, 1994.

[11] P. Deheuvels, "The caracterisation of distribution by order stat. and records value," Journal of Applied Probability, vol. 21, no. 2, pp. 326-334, 1984.

[12] P. Deheuvels, Majoration et minoration presque sûre optimale des éléments de la statistique ordonnée d'un échantillon croissant de variables aléatoires indépendantes, Nota Presentata dal Socio. Staraneno A. Lichnerowicz, 1974.

[13] O. Barndorff-Nielsen, "On the limit behaviour of extreme order statistics," Annals of Mathematical Statistics, vol. 34, pp. 992$1002,1963$. 


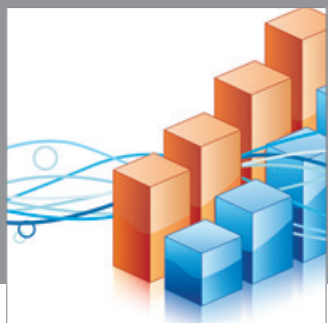

Advances in

Operations Research

mansans

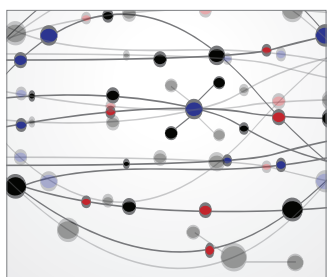

The Scientific World Journal
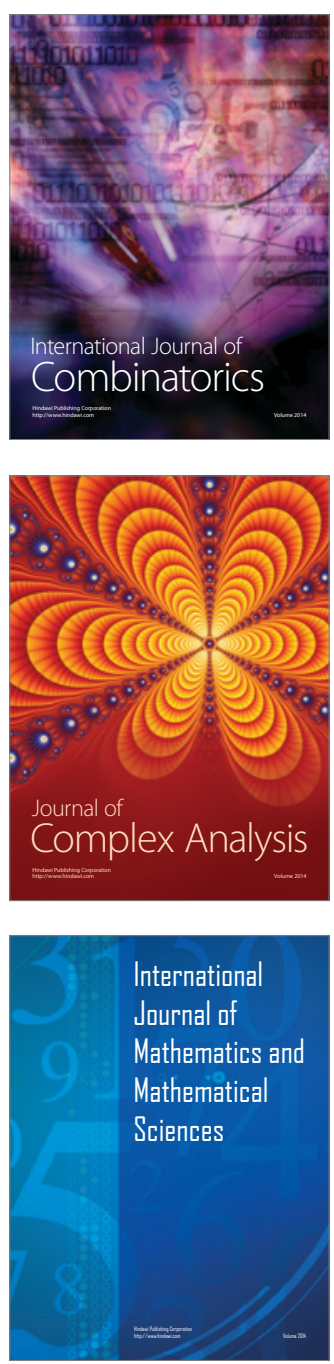
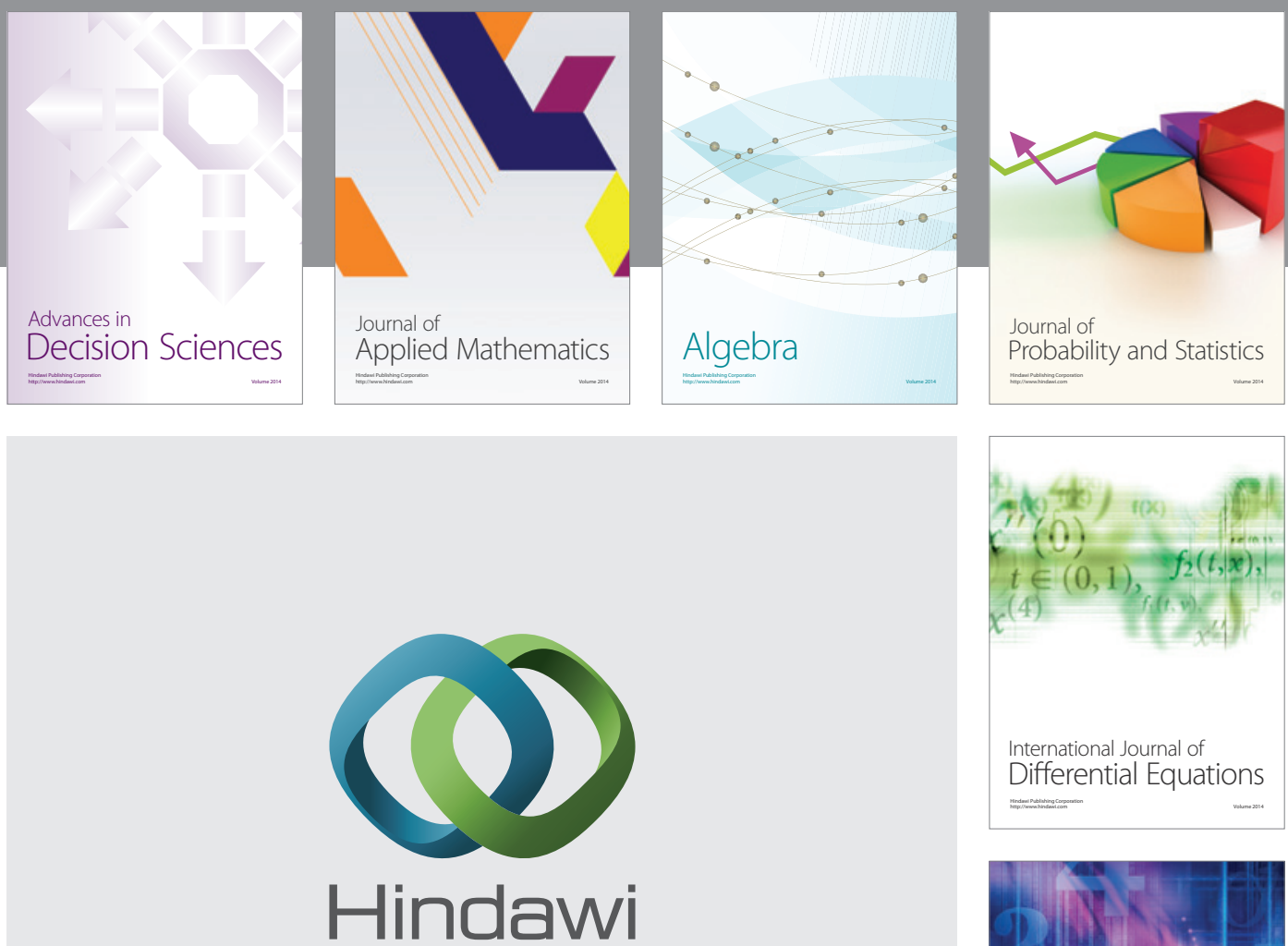

Submit your manuscripts at http://www.hindawi.com
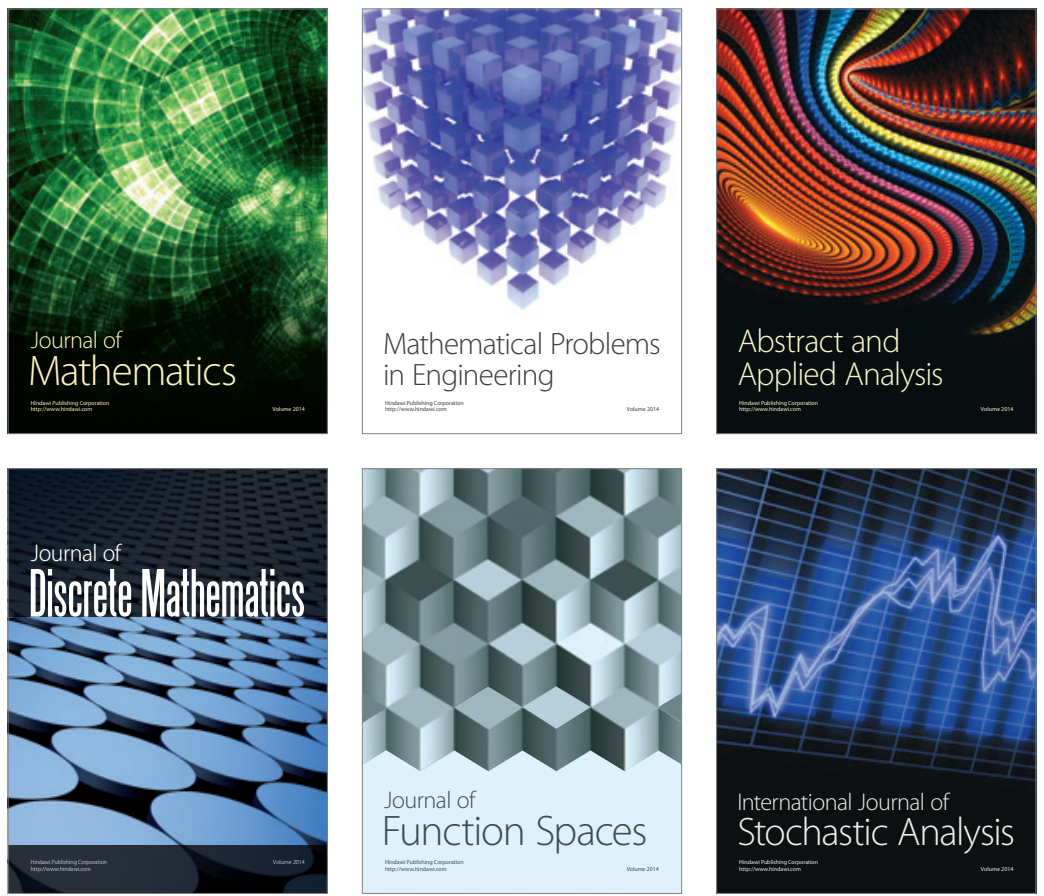

Journal of

Function Spaces

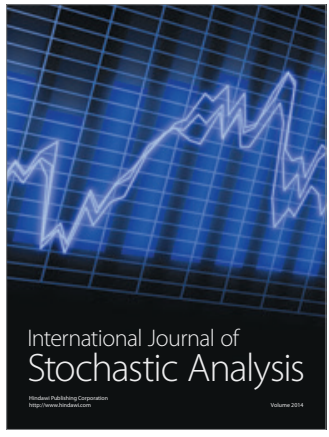

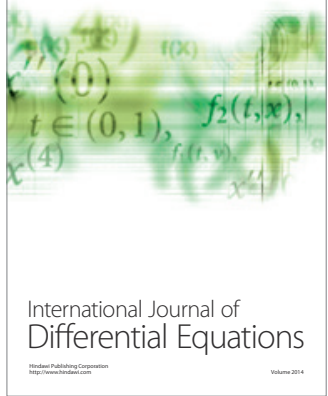
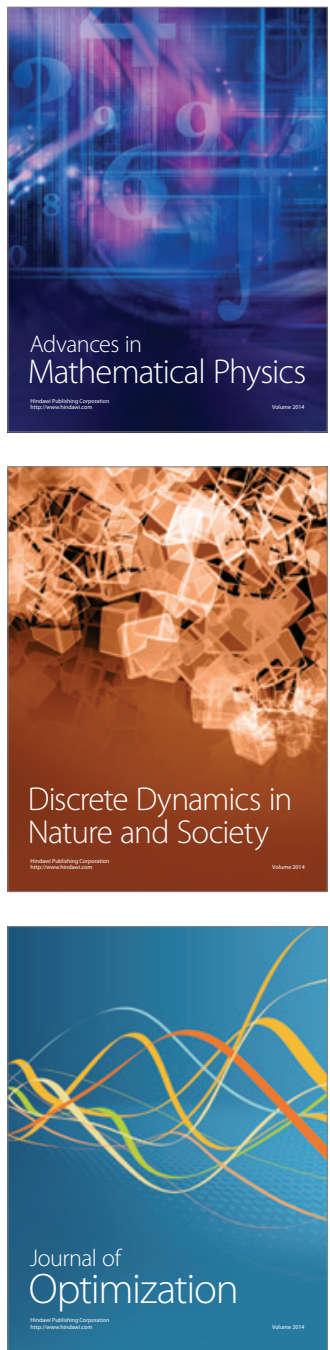